\title{
STUDIES ON THE DIFFERENTIAL EQUATIONS OF ENZYME KINETICS. II. BIMOLECULAR REACTION: THE FITTING OF EXPERIMENTAL DATA TO THE SIMPLIFIED MODEL.
}

\section{ClaUde maRMASSE}

\author{
Biophysics Laboratory \\ La Feria, Texas 78559-0951 \\ and \\ JOSEPH WIENER \\ Department of Mathematics \\ Pan American University \\ Edinburg, Texas 78539 \\ (Received April 29, 1987)
}

ABSTRACT. Efficient and unbiased estimates of the parameters of the differential system, as well as simultaneous fiducial limits, are obtained through an (eventually weighted) least-squares fitting to a Taylor expansion of the concentration of the products of the reaction.

ISEY WORDS AND PHRASES. Enzyme Kinetics, Differential Equations, Fitting. 1980 MATHEMATICS SUBJECT CLASSIFICATION CODES. 34A45, 80A30, 65D30.

\section{INTRODUCTION.}

Let us consider a reaction in which an enzyme $E$ reacts with a substrate $S$; this results in the formation of an activated complex Es* which, in the simplified model under investigation, splits irreversibly into the enzyme and the products of the reaction $P$. The corresponding chemical scheme is

$$
\mathrm{E}+\mathrm{S} \underset{\mathrm{k}_{-1}}{\stackrel{\mathrm{k}_{1}}{\rightleftharpoons}} \mathrm{ES}{ }^{*} \stackrel{\mathrm{k}_{2}}{\longrightarrow} \mathrm{E}+\mathrm{P},
$$

where the $k^{\prime} s$ are velocity constants. Let $e, s$ and $x$ be the concentration of free enzyme, free substrate and activated complex respectively. Then the differential system which describes the kinetics of the reaction is

$$
\begin{aligned}
& \mathrm{ds} / \mathrm{d} t=-k_{1} e_{0} s+k_{-1} \mathrm{x}+\mathrm{k}_{1} s x \\
& \mathrm{dx} / \mathrm{dt}=\mathrm{k}_{1} \mathrm{e}_{\mathrm{o}} \mathrm{s}-\left(\mathrm{k}_{-1}+\mathrm{k}_{2}\right) \mathrm{x}-\mathrm{k}_{1} \mathrm{sx},
\end{aligned}
$$

where $e_{0}$ denotes the initial concentration of enzyme and with the initial conditions

$$
s(0)=s_{0}, \quad x(0)=0 \quad \text {. }
$$

This system is non-linear and no close-form solution is available. It has been 
snown in [1] that of the two classical approximations of $(1.1)-(1.2)$, that due to Haldane and Briggs and known as the steady-state approximation is preferable, but its domain of validity is limited to very small values of $e_{0}$ or $s_{0}$, which points to the need of a more direct approach. Anyway this approximation allows one to estimate only the two combinations of parameters $k_{2} e_{0}$ (the $v_{\max }$ of classical enzyme kinetics theory) and the Michaelis constant $k_{M}=\left(k_{-1}+k_{2}\right) / k_{1}$. Thus, even assuming that the Briggs-Haldane scheme would be used in conjunction with the extrapolation technique suggested in [1] and therefore made more reliable, it remains that it is intrinsically limited and leads to discard much of the information contained in a kinetic curve.

In practice the interest is focused on the initial portion of this curve because - a feature not restricted to experiments carried out with enzymes - parasite reactions may occur later and mask the phenomenon under scrutiny. In the BriggsHaldane method (and in the Michaelis-Menten one as we11) one uses exclusively the linear segment of the graph reaction velocity versus time. However the information sought, i.e. the numerical value of the parameters of the differential system is contained in part in the non-1inear portion of the curve and it is there generally speaking - that is whatever may be the dependent variable selected - that the analysis must be extended.

Thus the purpose of this paper is to describe a technique suitable for the fitting of a set of experimental data to the solution of (1.1)-(1.2) and for obtaining estimates, with their fiducial limits, of the parameters $e_{0}$ and $k^{\prime} s$. The discussion is conducted in terms of quantities easily evaluated by a digital computer.

\section{THE FUNDAMENTAL REGRESSION.}

Let there be $t_{i}(i=1,2, \ldots, n)$ the times at which a certain variable is monitored in order to define the kinetic curve of an enzymatic reaction. As usual we take $t$ as the (statistically) independent variable; we select as dependent variable the concentration of the products of the reaction $p$, which satisfies

$$
d p / d t=-(d s / d t+d x / d t)=k_{2} x
$$

Taking into account the initial conditions $p(0)=x(0)=0$ one obtains, near the origin, $p$ in the form

$$
p=k_{2}\left[x_{0}^{\prime} t^{2} / 2 !+x_{0}^{\prime \prime} t^{3} / 3 !+\ldots+x_{0}^{(i)} t^{i+1 /(i+1) !+\ldots], ~, ~}\right.
$$

where the' indicate derivatives with respect to time, and the problem is reduced to the evaluation of $x_{0}^{(i)}(i=2,3, \ldots)$. The graph of $p$ versus $t$ starts tangentially to the time axis (at the origin), with an upward concavity (as at the origin $\mathrm{dx} / \mathrm{dt}=$ 
$\mathrm{k}_{1} \mathrm{e}_{\mathrm{o}} \mathrm{s}_{\mathrm{o}}>0$ ) and later exhibits an inflexion point (at the time $\mathrm{T}$ of [1]). Thus, at the beginning of the reaction at least, the function $p(t)$ is strongly non-linear and therefore presents the features required for the analysis under consideration.

In order to facilitate the computer processing of the equations to be deve1oped, we relabel the parameters as follows,

$\begin{array}{llllll}\text { former notation: } & \mathrm{e}_{\mathrm{o}} & \mathrm{k}_{1} & \mathrm{k}_{-1} & \mathrm{k}_{2} \\ \text { new notation }: & \mathrm{k}_{1} & \mathrm{k}_{2} & \mathrm{k}_{3} & \mathrm{k}_{4}\end{array}$

Then (1.1) becomes

$$
\begin{aligned}
& \mathrm{ds} / \mathrm{dt}=\mathrm{K}_{3} \mathrm{x}-\mathrm{K}_{2}\left(\mathrm{~K}_{1}-\mathrm{x}\right) \mathrm{s} \\
& \mathrm{dx} / \mathrm{dt}=\mathrm{K}_{2}\left(\mathrm{~K}_{1}-\mathrm{x}\right) \mathrm{s}-\left(\mathrm{K}_{3}+\mathrm{K}_{4}\right) \mathrm{x}
\end{aligned}
$$

The solution $\mathrm{p}=\mathrm{p}\left(\mathrm{t} ; \mathrm{K}_{1}, \mathrm{~K}_{2}, \mathrm{~K}_{3}, \mathrm{~K}_{4}\right)$ is obtained by means of the standard Taylor expansion method. The calculations are much simplified if, taking into account (2.1), one rewrites $(2.2)$ in the non-canonical form

$$
\begin{aligned}
& s^{\prime}=K_{3} x-k_{2}\left(k_{1}-x\right) s \\
& x^{\prime}=-K_{4} x-s^{\prime} .
\end{aligned}
$$

Putting

$$
\begin{aligned}
& a=K_{1} K_{2}+K_{2} s_{o}+K_{3} \\
& b=a+K_{4} \\
& c=K_{1} K_{2}\left(2 K_{2} s_{0}-K_{4}\right) \\
& d=b^{2}+2 c+4 K_{1}^{2} K_{2} s
\end{aligned}
$$

one obtains a Taylor approximation in the form

$$
\mathrm{p}=\mathrm{K}_{1} \mathrm{~K}_{2} \mathrm{~K}_{4} \mathrm{~s}_{\mathrm{o}}\left[\mathrm{t}^{2} / 2 !-b t^{3} / 3 !+\left(\mathrm{b}^{2}+\mathrm{c}\right) \mathrm{t}^{4} / 4 !-\left(\mathrm{bd}-3 \mathrm{~K}_{1} \mathrm{~K}_{2}^{2} \mathrm{~K}_{4} \mathrm{~s}_{0}\right) \mathrm{t}^{5} / 5 !\right]
$$

Assume now that we know a set of approximate values $k_{i}(i=1,2,3,4)$ of the parameters. The effect of small variations $\Delta \mathrm{K}_{i}$ around these values on $p$ considered as a function of the $K_{i}$ 's is given by

$$
\mathrm{p}\left(\mathrm{t} ; \mathrm{K}_{1}+\Delta \mathrm{K}_{1}, \mathrm{~K}_{2}+\Delta \mathrm{K}_{2}, \mathrm{~K}_{3}+\Delta \mathrm{K}_{3}, \mathrm{~K}_{4}+\Delta \mathrm{K}_{4}\right)=\mathrm{p}\left(\mathrm{t} ; \mathrm{K}_{1}, \mathrm{~K}_{2}, \mathrm{~K}_{3}, \mathrm{~K}_{4}\right)+\sum_{i=1}^{4} \phi_{i} \Delta \mathrm{K}_{i}+\sum_{i=1}^{4} 0\left(\Delta \mathrm{K}_{i}^{2}\right)
$$

in the domain D discussed in Section 5 and with $\phi_{i}=\frac{\partial p}{\partial K_{i}}(i=1,2,3,4)$. We have

$$
\begin{aligned}
\phi_{1} / K_{2} K_{4} s_{o}= & t^{2} / 2 !-\left(b+K_{1} K_{2}\right) t^{3} / 3 !+\left(b+2 b K_{1} K_{2}+2 c\right) t^{4} / 4 ! \\
& -\left[\left(b+K_{1} K_{2}\right) d+2 b\left(b K_{1} K_{2}+c+2 K_{1} K_{2}^{2} s_{o}\right)-6 K_{1} K_{2}^{2} K_{4} s_{0}\right] t^{5} / 5 ! \\
\phi_{2} / K_{1} K_{4} s_{o}= & t^{2} / 2 !-\left(a+b-K_{3}\right) t^{3} / 3 !+\left[b^{2}+2 b\left(a-K_{3}\right)+2 c+2 K_{1} K_{2}^{2} s_{0}\right] t^{5} / 4 ! \\
& -\left\{\left(a+b-K_{3}\right) d+2 b\left[\left(a-K_{3}\right) b+c+6 K_{1} K_{2}^{2} s_{0}\right]-9 K_{1} K_{2}^{2} K_{4} s_{0}\right\} t^{5} / 5 ! \\
\phi_{3} / K_{1} K_{2} K_{4} s_{0}= & -t^{3} / 3 !+2 b t^{4} / 4 !-\left(2 b^{2}+d\right) t^{5} / 5 ! \\
\phi_{4} / K_{1} K_{2} s_{0}= & t^{2} / 2 !-\left(b+K_{4}\right) t^{3} / 3 !+\left(b^{2}+2 b K_{4}+c-K_{1} K_{2} K_{4}\right) t^{4} / 4 ! \\
- & {\left[\left(b+K_{4}\right) d+2 b K_{4}\left(b-K_{1} K_{2}\right)-6 K_{1} K_{2}^{2} K_{4} s_{0}\right] t^{5} / 5 ! }
\end{aligned}
$$


Given a certain set of experimental values $p_{i}^{\exp }(i=1,2, \ldots, n)$, one can apply the classical Gauss linearization technique and compute, within the domain D, corrections $\Delta K_{i}(i=1,2,3,4)$ to the estimates $K_{i}$ by means of the quadrilinear regression $\mathrm{p}^{\exp }=\mathrm{p}\left(\mathrm{t} ; \mathrm{K}_{1}, \mathrm{~K}_{2}, \mathrm{~K}_{3}, \mathrm{~K}_{4}\right)+\sum_{i=1}^{4} \phi_{1} \Delta \mathrm{K}_{1}$

This is a constrained regression as the coefficient of $p$ is equal to unity. In order to remove this constraint we define the new variable $z \equiv p^{e x p}-p$ and relation (2.4) becomes

$$
z=\sum_{i=1}^{4} \phi_{i} \Delta K_{i}
$$

The preceding regression constitutes the fundamental regression of the problem. $f_{i}-i 1$ be noticed that it is mathematically constrained (as opposed to statistical1y) to pass through the origin. Hence there remain $n-4$ degrees of freedom.

\section{ESTIMATES AND FIDUCIAL LIMITS: BASIC THEORY.}

We first assume that $\mathrm{p}^{\exp }$ is normally distributed. Then a least-squares solution of (2.5) or more generally of a N-1inear regression of the same type will furnish estimates of $\Delta K_{i}(i=1,2, \ldots, N)$ which are both efficient and unbiased. Hence by solving iteratively the regression until the increments are made sufficiently sma11, one will obtain efficient and unbiased estimates of the $\mathrm{K}_{i}$ 's. Denoting by $\phi_{i k}$ and $z_{k}$ respectively the value of $\phi_{i}$ and $z$ for the $k$-th experimental point, the normal equations of the problem can be written as $\mathrm{T} \vec{\Delta}=\vec{q}$, where $\mathrm{T}$ is the real symmetric matrix of general element

$$
t_{i j}=\sum_{k=1}^{n} \phi_{i k} \phi_{j k} \quad(i, j=1,2, \ldots, N)
$$

$\triangle$ the vector of general element $\Delta \mathrm{K}_{i}$ and $\vec{q}$ the vector of general element

$$
q_{i}=\sum_{k=1}^{4} \phi_{i k} z_{k} \quad(i=1,2, \ldots, N)
$$

Then one formally obtains $\vec{\Delta}$ from $\vec{\Delta}=\mathrm{T}^{-1} \overrightarrow{\mathrm{q}}$.

The total sum of squares is $\Sigma z_{k}^{2}$ and the sum of squares accounted for by the regression is $\vec{\Delta} \cdot \vec{q}$. Hence the variance $s^{2}$ is estimated from

$$
(n-N) s^{2}=\sum_{k=1}^{n} z_{k}^{2}-\vec{\Delta} \cdot \vec{q} \text {. }
$$

Let $t^{i j}$ be the general element of $T^{-1}$. Then the variance of $k_{i}$ is $s^{2} t^{i i}$ and as $\mathrm{K}_{\mathrm{i}}$ is normally distributed, fiducial limits for this quantity (and consequently for $k_{i}$ ) can be obtained as

$$
\mathrm{K}_{\mathrm{i}}+\mathrm{Str} \mathrm{t}^{\mathrm{ii}} \quad(\mathrm{i}=1,2, \ldots, \mathrm{N}) \quad,
$$

where $t$ is the value of the Student $t$-distribution at the selected level of confidence.

One could use the fiducial limits (3.2) to test statistically the concordance between the set of experimental data and the computed values of the parameters, but 
such a test is inefficient because these limits are not simultaneous: the probability that they be reached simultaneously is smaller than the probability corresponding to the selected $t$ point [4].

The determination of simultaneous fiducial limits has already been treated in [2] and [3] but we give here a more streamlined derivation. The sum of squares accounted for by the regression $\vec{\Delta} \cdot \vec{q}$, used in (3.1) to estimate the variance, can be written as

$$
Q=\vec{\Delta}^{\prime} \mathrm{T} \vec{\Delta} \quad,
$$

where $a$ ' denotes a transposition; thus $Q$ is a quadratic form which is distributed as $\chi^{2}$ with $\mathrm{N}$ degrees of freedom. On the other hand $\mathrm{s}^{2}$ is $\chi^{2}$-distributed with $\mathrm{n}-\mathrm{N}$ degrees of freedom. Hence the ratio $(Q / N) \div\left[S^{2} /(n-N)\right]$ is distributed as $F$ with $N$ and $\mathrm{n}-\mathrm{N}$ degrees of freedom and the relation

$$
Q=[N /(n-N)] F S^{2}
$$

defines simultaneous limits for the $\Delta \mathrm{K}_{i}$ 's at the level of confidence selected for $F$.

In many situations however, the interest is focused on the relative variations $\Delta \mathrm{K}_{i} / \mathrm{K}_{i}$ rather than on the magnitude of the $\mathrm{K}_{i}$ 's. Therefore we define

$$
\alpha_{i}=\Delta K_{i} / K_{i} \quad(i=1,2, \ldots, N)
$$

and $Q$ becomes

$$
Q=\sum_{i=1}^{N} \sum_{j=1}^{N} t_{i j} \alpha_{i} \alpha_{j} K_{i} K_{j}
$$

In the space of the $\alpha_{i}$ 's the solutions of (3.4)-(3.5), that is feasible simultaneous limits, are hyperellipsoids and this makes in most cases for unnecessarily complicated calculations. Now amongst all these ellipsoids there is, at a given level of confidence, one hypersphere whose radius $\mathrm{x}$ satisfies

$$
x^{2}(\vec{K} \cdot T \vec{K})=[N /(n-N)] F^{2},
$$

where $\vec{K}$ is the vector of general element $k_{i}$. Let us define

$$
\mathrm{Q}_{\mathrm{K}}=\overrightarrow{\mathrm{K}}^{\prime} \mathrm{TK} \quad \text {; }
$$

it will be noticed that $Q_{K}$ is computed from quantities present in memory at the end of the iteration. Then (3.6) takes the form

$$
\mathrm{x}^{2} \mathrm{Q}_{\mathrm{K}}=[\mathrm{N} /(\mathrm{n}-\mathrm{N})] \mathrm{FS}^{2}
$$

Let $x_{1}$ be the value of $x$ for the critical value $F=1$. Thus

$$
\mathrm{x}_{1}^{2} \mathrm{Q}_{\mathrm{K}}=[\mathrm{N} /(\mathrm{n}-\mathrm{N})] \mathrm{s}^{2}
$$

and simultaneous relative departures of the parameters from the computed values $K_{i}$ by an amount no greater than $x_{1}$ are meaningless, whatever may be the level of confidence. This is to say that $x_{1}$ is a statistic which measures the degree of concordance between say the solution (2.3) of the differential system and the (normally dis- 
tributed) set of experimental data.

Now (3.6) can be rewritten as $x^{2}=x_{1}^{2} F$. Hence once $x_{1}$ is known, one can compute simultaneous fiducial limits at a soecified level of confidence.

When the variance of $p^{\exp }$ is not homogeneous, it is sufficient to divide both sides of $(2.5)$ by the weight factor $w=\operatorname{Var}\left(p^{\exp }\right)$. The introduction of $w$ is quite essential: for example when the course of the reaction is followed spectrophotometrically, as in that case the variance of $p^{\exp }$ exhibits dramatic variations on the domain of absorbancies most used[5]. For the calculation of $w$ in situations of practical interest, see [3].

Finally it must be emphasized that even when one exclusively analyzes sets of normally distributed pexp, it is advisable to introduce formally the weight factors $\mathrm{w}$ (a11 equal to 1 when the variance is homogeneous) because the scanning technique described in [3] and used to detect inhomogeneities in a set of data analyzed with respect to a steady-state approximation formula is directly applicable to the case at hand. Assume that the $\mathrm{k}$-th point is aberrant: if one depresses in turn the influence of each point by increasing its associated $w$ factor, the $x_{1}$ values calculated will be more or less of the same magnitude, except for the $k-t h$ one which will be markedly smaller. One can then test the hypothesis that the $k-t h$ point is the only aberrant one of the set in the following way: the influence of that point is depressed or quasi-eliminated by increasing its $w$ factor and the remaining points are scanned as previously. If the sole $k$-th point stands apart from the others, the $x_{1}$ values recorded now will have more or less the same magnitude; etc.

\section{THE ILL-CONDITIONED CASE.}

By construction the matrix $T$ is positive definite, but barely so in fact, and it is nearly singular (as can be verified, e.g., on the system $s_{0}=100, k_{1}=k_{2}=.01$, $\left.\mathrm{K}_{3}=.005, \mathrm{~K}_{4}=.008\right)$. On the other hand it must be emphasized that to some extent the condition of a linear system is relative, in the sense that, as in any linear algebra computation, the machine word size and the presence or the absence of a doublelength accumulator are of paramount importance. Furthermore, even if the matrix of the complete system, that is to say with $N=4$ variables, cannot be inverted, it may happen that through the exclusion of one variable, the matrix of order 3 so obtained will prove readily invertible on the particular computer used. For instance the condition of the matrix $\mathrm{T}$ associated with the system mentioned improves when one excludes either $K_{1}$ or $K_{2}$. Such a feature could obviously be used in special circumstances to bracket by successive approximations satisfactory estimates of the 4 para- 
meters but in general it is advisable to handle directly the 4-parameters problem and because from a practical standpoint the most useful discussion refers to a worst case situation, we shall examine the analysis of the solution (2.3) of the same example within the domain D (see Section 5), with a machine using mantissae corresponding to 13-14D (a value typical of many a microprocessor-based system); all the operations were performed in single length mode.

The most striking feature of the matrix $\mathrm{T}$ is that it loses easily its positive definiteness, for example in the course of a Cholevsky triangularization. In fact the inversion by all the classical techniques involving a triangularization fails. Thus one must abandon any idea of inverting en bloc the matrix $T$; as a matter of fact this is of little consequence because the only computation in which the $t^{i j}-$ strictly speaking the $t^{i i}(i=1,2,3,4)$ - are involved in an essential way, is that of the variance of the parameters. But these variances are not much used as $x_{1}$ is a more convenient and realistic statistic. Thus one can dispense with the inversion of $T$ and we consider now the lesser problem of solving once the normal equations by an iterative technique which does not require a preliminary deep transformation of the matrix of the coefficients. The standard techniques for solving a linear system with a positive and non-singular matrix, such as the conjugate gradient method or the steepest descent method, all failed.

An obvious way to deal with the evanescence of the positive definiteness of $T$ is to multiply on the left the normal equations by $\mathrm{T}$ but the multiplication (here the squaring) of ill-conditioned matrices is usually deemed undesirable. This formal calculation of $\mathrm{T}^{2}$ is avoided in a variant described by Hestenes and Stiefe1 [6]. This procedure proved unsuitable for our needs because huge oscillations of the terms $b_{i}$ of formulae $(10: 2)$ of [6] were recorded, although the stability of the coefficients $a_{i}$ was acceptable. This suggests to modify the method and to recalculate at each step the residual vector $\vec{r}$ from its definition.

This is to say that we use the following algorithm:

$$
\begin{aligned}
& \overrightarrow{\mathbf{r}}=\overrightarrow{\mathrm{q}}-\mathrm{T} \vec{\Delta} \\
& \overrightarrow{\mathbf{u}}=\mathrm{T} \overrightarrow{\mathrm{r}} \\
& \mathrm{a}=|\mathrm{Tr}|^{2} /|\mathrm{T} \overrightarrow{\mathrm{u}}|^{2} \\
& \vec{\Delta}_{\text {new }}=\vec{\Delta}_{\text {old }}+\mathrm{au}
\end{aligned}
$$

with the initial value $\vec{\Delta}=0$. No stability problem was encountered.

This procedure bears little resemblance to a conjugate gradient technique, which is essentially an orthogonalization method. On the other hand the first step of 
the conjugate gradient method and that of the steepest descent method are identical [7]; thus the algorithm (4.1) is best classified as that of an iterated steepest descent and the general theory of the steepest descent method [7] is applicable to each step individua11y.

The iterated steepest descent method shares with all iterative techniques one feature, viz. there comes a time when the limiting accuracy is reached, which implies in turn the existence of a limiting accuracy for the estimates of the parameters. The limiting accuracy of the normal equations routine is easily observable in the case at hand; hence an efficient strategy (worst case situation) is to subordinate the whole computation, including the exit from the fundamental regression iteration, primarily to the limiting accuracy exhibited by the normal equations routine rather than set beforehand strict escape clauses of the type $\left|\Delta \mathrm{K}_{i}\right|$ and or $\left|\Delta \mathrm{K}_{i}\right| / \mathrm{K}_{i}<10^{-3}-10^{-6}$, $(i=1,2,3,4)$.

Needless to say the exclusion of one variable may result in an appreciable improvement of the limiting accuracy.

\section{DISCUSSION.}

There remains to characterize the domain $\mathrm{D}$. It will be noticed that $\mathrm{K}_{1}, \mathrm{~K}_{2}$ or $\mathrm{K}_{4}$ must not vanish as then the matrix $\mathrm{T}$ becomes singular. Furthermore negative values of the $K^{\prime} s$ are meaningless and therefore we take as first conditions for defining $D$ :

$$
\mathrm{K}_{1}, \mathrm{~K}_{2}, \mathrm{~K}_{4}>0 \quad \mathrm{~K}_{3} \geq 0
$$

The conditions of validity of the expansion (2.3) are the same as those of $x(t)$ and $s(t)$. In order to study the latter we consider system (2.2) in the rectangle

$$
\left|s-s_{0}\right| \leqq B \quad, \quad|x| \leqq B \quad,
$$

where $B=\max \left(s_{0}, K_{1}\right)$. Let $f_{1}(s, x)$ and $f_{2}(s, x)$ denote the right-hand sides of (2.2); then we have $[$ in (5.2)] the following estimates:

$$
\begin{aligned}
& \left|f_{1}(s, x)\right| \leqq K_{3} B+K_{2}\left(K_{1}+B\right)\left(s_{0}+B\right) \\
& \left|f_{2}(s, x)\right| \leqq K_{2}\left(K_{1}+B\right)\left(s_{0}+B\right)+\left(K_{3}+K_{4}\right) B
\end{aligned}
$$

Putting

$$
M=K_{2}\left(K_{1}+B\right)\left(s_{0}+B\right)+\left(K_{3}+K_{4}\right) B
$$

we have in (5.2)

$$
\left|f_{1}(s, x)\right| \leqq M \quad, \quad\left|f_{2}(s, x)\right| \leq M
$$

Therefore the problem (1.2)-(2.2) has a unique solution defined on the inter-

val

$$
|t|<B / M
$$

Since $f_{1}(s, x)$ and $f_{2}(s, x)$ are analytic (and even entire) functions, the solution 
$\{s(t), x(t)\}$ is analytic on (5.3). Hence an upper bound of the maximum sampling time allowed $t_{\max }=\mathrm{B} / \mathrm{M}$ is obtained as

$$
t_{\max }=B /\left[K_{2}\left(K_{1}+B\right)\left(s_{0}+B\right)+\left(K_{3}+K_{4}\right) B\right]
$$

Whatever may be the largest of the two quantities $s_{0}$ and $k_{1}$, one obtains

$$
\mathrm{t}_{\max }=\left[2 \mathrm{~K}_{2}\left(\mathrm{~s}_{0}+\mathrm{K}_{1}\right)+\mathrm{K}_{3}+\mathrm{k}_{4}\right]^{-1}
$$

In other words $t_{\max }$ satisfies

$$
\left(a+b-k_{3}\right) t_{\max }=1, \quad,
$$

and the domain $\mathrm{D}$ is characterized by $(5.1)-(5.4)$.

The practical implementation of the techniques discussed in this paper and their implications regarding the experimental design of enzyme kinetics measurements will be described elsewhere. Suffice it to point out here that an immediate consequence and a major advantage of this numerical procedure is that it furnishes an estimate of $e_{0}\left(=k_{1}\right)$ in the same units as those used for $s_{0}$. In other words, through the analysis of a kinetic curve one can obtain directly the value of the initial concentration of an enzyme in wel1-defined physical units.

ACKNOWLEDGMENT: Research partially supported by U.S. Army Grant IDAAG2@ $24 . ?-0034$. ACKNOWLEDGMENT: Research of the second author was partially supported by U.S. Army Research Grant DAAG29-84-G-0034.

\section{REFERENCES .}

1. MARMASSE, C. and WIENER, J., Studies on the differential equations of enzyme kinetics. I. Bimolecular reaction: simplified model, Internat. J. Math. \& Math. Sci., 1986, in print.

2. MARMASSE, C., Structure analysis of physical data. I. General theory, Rev. Mex. Fis., 20 (1971), 239-52.

3. MARMASSE, C., Enzyme kinetics - Physical bases, data analysis and uses, Gordon and Breach, New York, 1977.

4. WILliAMS, E., Regression analysis, John Wiley \& Sons, New York, 1959.

5. MARMASSE, C., On the numerical exploitation of spectrometric data, App1. Opt., $\underline{4}(1965), 1632-3$

6. HESTENES, M.R. and STIEFEL, E., Methods of conjugate gradients for solving linear system, J. Res. Nat. Bur. Standards, 49 (1952), 409-38.

7. HOUSEHOLDER, A.S., Principles of numerical analysis, McGrawHi11, New York, 1953. 


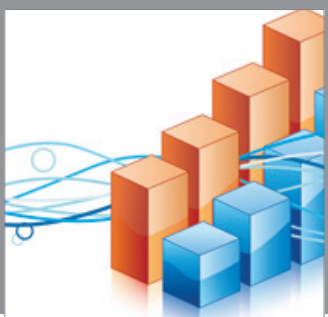

Advances in

Operations Research

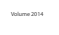

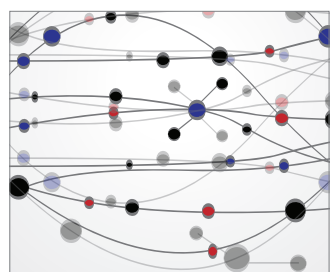

\section{The Scientific} World Journal
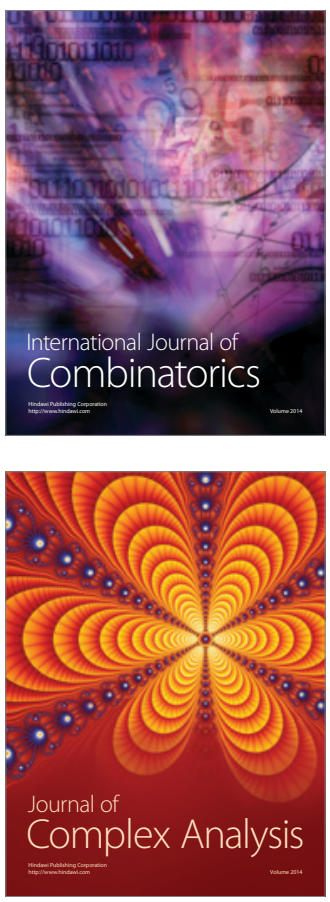

International Journal of

Mathematics and

Mathematical

Sciences
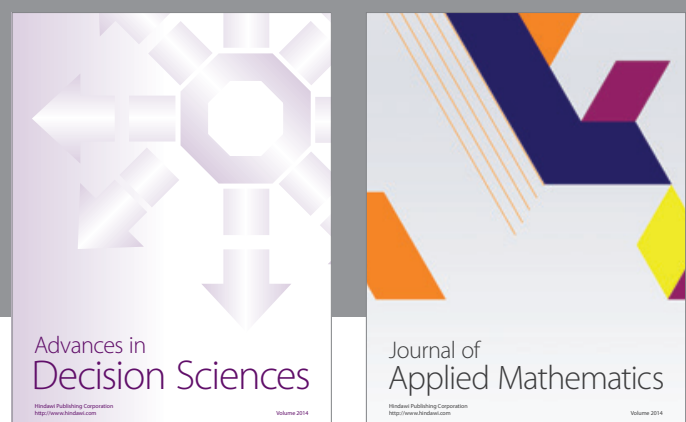

Journal of

Applied Mathematics
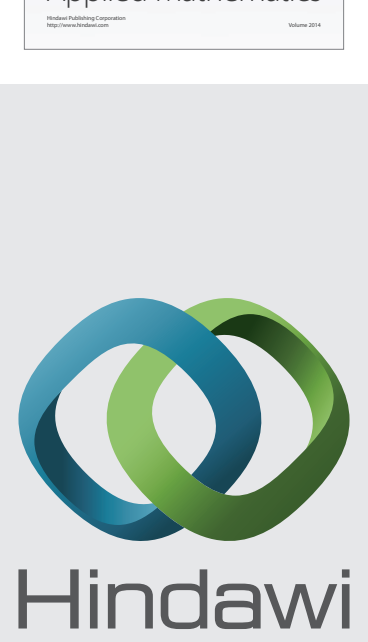

Submit your manuscripts at http://www.hindawi.com
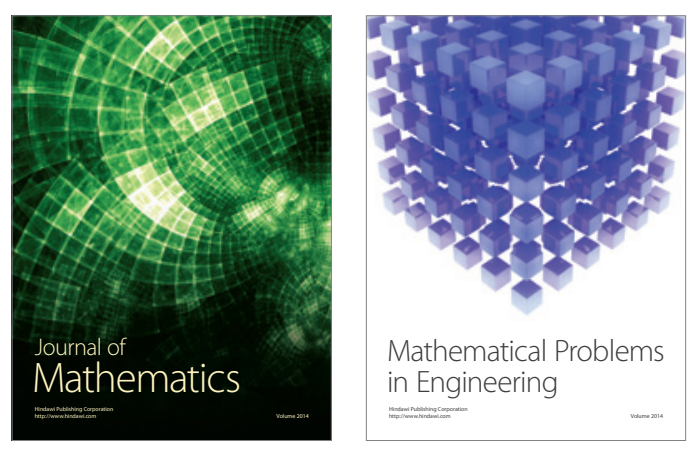

Mathematical Problems in Engineering
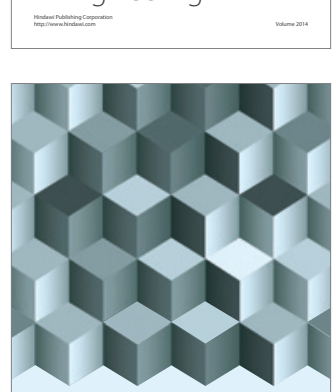

Journal of

Function Spaces
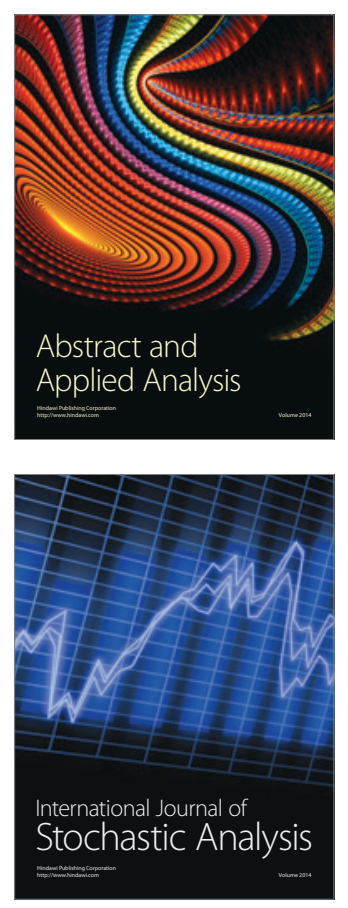

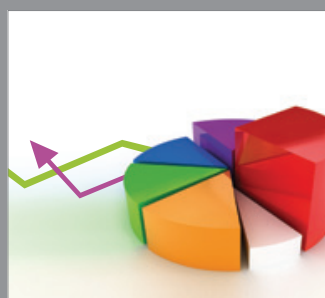

ournal of

Probability and Statistics

Promensencen
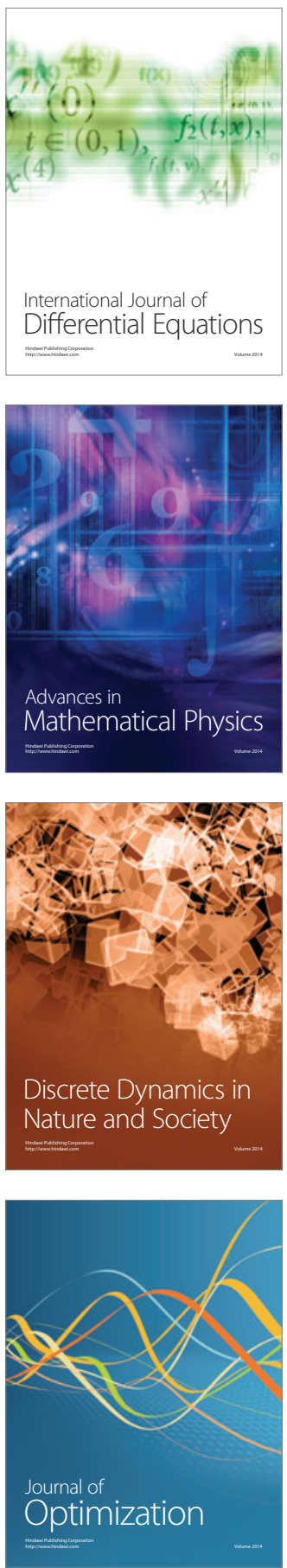